\title{
Traducción de fragmentos de "La Divina Comedia"*
}

\author{
por
}

Manuel Beltroy

\author{
P R E S E N T A C I O N
}

LA TRADUCCION castellana de algunos fragmentos de Tres Cantos del inmortal Poema del más grande de los Poetas Italianos y Europeos que me honro en presentar a la Facultad de Letras y Ciencias Humanas de mi Alma Mater de San Marcos y a la Ilustre Embajada de Italia en nuestro Pais, con ocasión del VII Centenario del Nacimiento de tan excelso Poeta y como pequeña contribución mía y del Claustro al Homenaje que el mundo entero le tributa y en su Genio al de la Insigne Nación Italiana, es ofrenda de profunda devoción literaria y tributo de ferviente amor y admiración a la Gran Madre Itálica y al egregio Hijo Florentino.

En verdad, nunca hubiera osado emprender Sesta mínima versión si no me hubieran movido a ello $\mathrm{mi}$ larga frecuentación de la vida y la obra del soberbio Gibelino y el amor admirativo que aquéllas me inspiraron, desde los días de $\mathrm{mi}$ ya lejana adolescencia, en que tuve la fortuna de conocerlas.

Fue el caso que, habiendo elegido como presente maternal, cuando aún era colegial, la hermosa edición de la sublime Epopeya publicada por la famosa Casa Editora catalana Montaner Simón, que presentara la acabada traducción española en prosa del texto original, de Don Cayetano Rosell, juntamente con éste, acompañada por las magníficas ilustraciones de Gustavo Doré, en que el genial dibujante se eleva a la altura del supremo vate de Florencia, en aquella, esplén-

\footnotetext{
* Esta fina versión -que Manuel Beltroy alcanzó a ver impresa, pocos meses antes de su muerte, en separata que sc ndelantó en homenaje a Dante- constituye el último trabajo universitario del maestro sanmarquino recientemente desaparecido. Entre las múltiples facetas de su actividad intelectual, destaca su labor de traductor de clásicos y modernos italianos y franceses, en las que puso al servicio de la transmisión de la cultura su capacidad y dotes de clicnz y delicaclo intérprete literario, en abnegado esfuerzo en pro del mejor conocimiento de las más altas figuras de la creación artística universal. Le rendimos en esta ocasión un tributo de reconocimiento y admiración por su labor de maestro y virtuoso de las letras.
} 
dida edición, se me abrieron las puertas, a la par, de la incomparable creación dantesca y de la magna lengua italiana.

Aprendi, pues. en esa Obra Maestra, a conocer al más alto Creador Poético de su Patria y de Europa al mismo tiempo que bebía en esa óptima fuente la clara linfa del lenguaje italiano, y, por puro placer literario, sin otra mira interesada, me puse a leer, y traducir, en són de amateur, los pasajes que más me gustaron de la Divina Comedia.

$\mathrm{Si}$ he escogido los tres fragmentos de los tres Cantos del Infierno que con su texto original presento en este opuisculo ha sido más bien llevado Dor un motivo estético que por el hondo significado filosópico y humano que encierran los temas de aquellos pasajes y en que parecen compendiarse y definirse tres aspectos capitales de la personalidad dantesca y de su interpretación del mundo y de la vida: en el encuentro con Virgilio, el amor a la sabiduria, que le conduce al amor de Beatriz y de Dios; en el coloquio con Francesca, su pasionalidad amorosa, clave de su temperamento y su carácter; y en el episodio trágico del Conde Ugolino, su pasión por la justicia y su indomable rectitud politica.

En mi muy modesta versión he procurado ceñirme al canon que ha guiado constantemente mi labor de traductor: conciliar la literaliclad con la literariedad, es decir, toda la fidelidad posible al texto del autor con su transubstanciación al espiritu de la lengua y la literatura de nuestra gente.

Sírvame, así, de excusa y explicación para esta traslación, el famoso disiico dantesco:

Vagliami il fungo studio e il grande amore
che m'han fatto cercar lo tue volume.

MANUEL BELTROY

Catedrático Emérito y Ex-Catedrático de la Facultad de Letras 


\title{
A D ANTE
}

En el Séptimo Centenario de su Nacimiento.

\begin{abstract}
"Tú Guía, Tú Señor y Tú Maestro" llamaste al Vate egregio Mantüano, $y$ tí eres también Gran Doctor nuestro, Señor excelso y Guia soberano.

Tú que, en virtud de tu latino ancestro, transfiguraste en divinal lo humano;

y con la luz radiante de tu estro iluminaste el Mundo y el arcano.

Ti que con sangre vivida escribiste $y$ por eso en espiritu pudiste sublimar à la triste Humanidad,

Vive por siempreien lsobrehümana gloria, por encima del Tiempo y de la Historia, en el Cielo de tu Inmortalidad.
\end{abstract}

MANUEL BELTROY.

Lima, Mayo de 1965. 
$L A D I V I N A$ COMEDIA

$$
\begin{array}{llllllll}
I & N & F & E & R & N & O
\end{array}
$$

\section{CANTO PRIMO}

NEL mezzo del cammin di nostra vita mi ritrovai per una selva oscura, che la diritta via era smarrita.

Ahi quanto a dir qual era cosa dura questa selva selvaggia ed aspra e forte che nel pensier rinnova la paura!

Tanto é amara che poco é piu morte: ma per trattar del ben ch'ivi trovai diro dell'altree cose ch'io v'ho scorte.

I'non so ben ridir com'io v'entrai, Tant'era pien di sonno in su quel punto che la verace via abbandonai.

Ma po'ch'io fui al pie d'un colle giunto la ove terminava quella valle che m'avea di paura il cor compunto,

Guardai in -alto, e coidita sue spalle vestite gia de'raggi debl pianeta che mena dritto altrui per ogni calle.

Allor fu la paura un poco queta che nel lago del cor m'era durata la notte ch'i passai con tanta pieta.

$E$ come quei che con lena affannata uscito fuor del pelago alla riva si volge all'acqua perigliosa e guata,

Cosi l'animo mio ch'ancor fuggiva, si volse'ndietro a rimirar lo passo che non lascio giammai persona viva.

Poi ch'ebbi riposato il corpo lasso ripresi via per la piaggia diserta, si che'l pie fermo sempre era'l piú basso. 


\section{CANTO PRIMERO}

A mitad del camino de esta vida Halléme en medio de una selva obscura, ya que la via recta era perdida.

¡Cómo podré decir, ay, la amargura de esta selva salvaje, áspera y fuerte, que al pensarla renueva la pavura!

Tanto es de amarga que no es más la muerte; mas para hablar del bien que alli encontré diré lo que con él me envió la suerte.

No acierto a referir cómo alli entré, tan soñoliento fui en la hora mezquina en que la via vera abandoné.

Mas al llegar al pie de una colina en donde aquel valle terminaba que el corazón me hiriera con su espina.

Miré hacia larriba yz vi que se alumbraba con los rayos del astro que asegura la ruta de quienquier, libre $y$ sin traba.

Calmose entonce un poco la pavura que de mi sangre el mar habia agitado en la noche fatal de mi tristura.

$Y$ como aquel que al salir angustiado del oceáno hacia la playa esquiva vuélvese a ver el piélago agitado,

Asi el anima mia, aun fugitiva, volviose a ver de nuevo el paso duro que nunca abandonó persona viva.

Después de reposar de tal apuro segui el viaje por la playa desierta, sintiendo el pie inferior el más seguro. 
Ed ecco quasi al comminciar dell'erta una lonza leggiera e presta molto che di pel maculato era coperta;

E non mi si partia dinanzi al volto, anz'impediva tanto'l mio cammino ch'i'fui per ritonar piu volte volto.

Temp'era del principio del mattino, e'l sol montava in su con quelle stelle ch'eran con lui quando l'amor divino

Mosse da prima quelle cose belle; si ch'a bene sperar m'eran cagione di quella fera la gajetta pele,

L'ora del tempo e la dolce stagione; ma non si che paura non mi desse la vista che m'apparve d'un leone.

Questi parea che contra me venesse con la testa alta e con rabbiosa fame, si che parea che laer no temesse.

Ed una lupa che di tutte brame sembiava carca nella sua magrezza, $e$ molte genti fe' gia viver grame.

Questa mi porse tanto Cai graveza con la paura che uscia li dua vista ch'i'perdei la speranza dell'altezza.

E quale e quei che volentieri acquista, e giugne'l tempo che perder lo face, che'n tutt'i suoi pensier piange e s'attrista;

Tal mi fece la bestia senza pace, che venendomi'ncontro, a poco a poco mi ripingeva lâ dove'l sol tace.

Mentre ch'i'rovinava in basso loco, dinanzi gli occhi mi si fu offerto chi per lungo silenzio parea fioco.

Quando vidi costui nel gran diserto: -Miserere di me-, gridai a lui, qualche tu sii od ombra od uomo certo. 
He aqui que al franquear la tierra yerta surgió pantera rápida y voraz

que con manchada piel era cubierta;

$Y$ no se me apartaba de la faz,

antes tanto obstruía mi camino

que intenté varias veces ir atrás.

Rayaba entonce el albor matutino

$y$ alzábase el sol con las estrellas

con que nació cuando el Amor divino.

Movio al principio aquellas cosas bellas;

serian, pues, favorable ocasion

la piel manchada de la fiera aquella,

La hora del tiempo y la dulce estación, mas no tánto que no me intimidase

la vista y la presencia de un león.

Pareciome que contra mi avanzase, con la testa alta $y$ hambre tan rabiosa que hasta el aire mismo intimidase.

Y una loba en tal grado codiciosa, a pesar de su insolita flacura, que a mucha gente torna desdichosa.

Esta me penetro de tal tristura con el pavor que csuilvista infundia "SO" que perdi la esperanza de la altura.

$Y$ como aquél que atesora a porfía $y$ cuanao el tiempo de perder le llega es presa de mortal melancolia,

Tal me ocurrio con la bestia andariega, que acorralánáome implacablemente me iba empujando donde el sol se aniega.

Mientras yo regresaba a la pendiente ante mi vi surgir un ser incierto que mudo parecia por lo silente.

Todo fué que lo vi en el gran desierto le imploré a voces: -Ten piedad de $m i$, ya seas sombra o bien un hombre cierto. 
Risposemi: - Non uomo, uomo gi fui

e li parenti miei furon Lombardi,

e Mantovani per patria ambedui.

Nacqui SUB JULIO, ancor che josse tardi. e vissi a Roma sotto'l buono Augusto al tempo degli Dei falsi e bugiardi.

Poeta fui, e cantai di quel giusto figliuol d'Anchise che venne da Troja, poiche'l superbo Ilion fu combusto.

Ma tu perche ritorni a tanta noja? perché non sali il äilettoso monte ch'é principio e cagion di tutía gioja?

-Or se'tu quel Virgilio e quella fonte che spande di parlar si largo fiume?risposi lui con vergognosa fronte.

O degli altri poeit onorel e lume, vagliami'l lungo studio e'l grande amore che m'han fatto cercar lo tuo volume.

Tu se'lo mio muestro e'l mio autore:

Tu se' solo colui da cui'io tolsi lo bello stile ch m'ha fatto onore.

Vedi la bestia per cui io mi volsi: ajutami da le $i$, famoso saggio, ch'ella mi fa tremar le venne e $i$ polsi.

-A te convien tener altro viaggio, -rispose poi che lagrimar mi videse vuoi campar d'esto loco selvaggio:

che questa bestia per la qual tu gride non lascia altrui passar per la sua via, ma tanto lo impedisce che l'uccide;

ed ha natura si malvaggia e ria che mai non empie la bramosa voglia, che dopo el pasto ha piú fame che pria. 
Respondiome: -No soy hombre; ya fui; y mi padre y mi madre eran lombardos

y ambos de Mantua, donde yo naci.

Naci de Julio en los dias ya tardos $\nu$ viví en Roma bajo el buen Augusto, cuando los dioses falsos y bastardos.

Poeta fú $y$ celebré a aquel justo hijo de Anquises que de Troya vino después que el grande Ilion fue combusto.

Mas, ¿por qué tornas a este desatino $y$ no subes al monte deleitoso que de toda alegría es el camino?

-iOh, tú eres Virgilio -ruboroso le contesté-, la caudalosa fuente del de elocuencia rio tan copioso!

Honor y luz de la apolinea gente, válgame el largo estudio y grande amor con que busqué tus libros, vehemente.

Tu eres mi maestro y mi escritor dilecto, el rinico en quien aprendi el bello estilo que es todo $m i$ honor.

Mira ta bestia porchuien me volvt. Acorreme, oh sapiente personaje, que ella me hace temblar de frenest.

-Debes seguir otra ruta en tu viajerepuso luego que miro mi llanto si este sitio dejar quieres salvaje.

Que aquesta fiera que te causa espanto a nadie deja pasar por su via, mas le da muerte. al impedirle tanto.

$Y$ tan perversa es su natura e impia que nunca sacia su hambre codiciosa $y$ tras comer por más presas porfía. 


\section{CANTO QUINTO}

Posci ch'io ebbi il mio dottore audito nommar le donne antiche $e$ i cavalieri, pietá mi vinse e fui guasi smarrito.

Io comminciai: -Poeta, volontieri parlerei a que'duo cine'nsieme vanno, $e$ pajon si al vento esser leggieri.

Ed egli a me: -Vedrai quando saranno piú presso a noi; e tu allor li prega per quell'amor ch'ei mena, e quei verranno.

Si tosto come'l vento a noi li piega, mossi la voce: -O anime affannate, venite a noi parlar, s'altri nol niega.

Quali colombe dal clisio chiamate, con l'ali aperte e ferme al dolce nido volan per l'aer dal voler portate;

Cotali uscir della schiera ov' é Dido, a noi venendo per l'aer maligno; si forte tu l'affetuoso grido.

-O animal grazioso e benigno che visitando vai per l'aer perso noi che tignemmo'l mondo di sanguigno;

Se fosse amico il Re dell'universo, noi pregheremmo lui per la tua pace, po'ch'hai pieta del nostro mal perverso.

Di quel ch'udire e che parlar vi piace noi udiremo e parlercmo a vui, mentre ch'l vento come fa si tace.

Siede la terra dove nata fui su la marina dove'l Po discende per aver pace co'seguaci sui.

Amor ch'al cor gentil ratto s'apprende presse costui della bella persona che mi fu tolta, e'l modo ancor m'offende.

Amor ch'a nullo amato amar perdona mi prese del costui piacer si forte che come vedi ancor non m'abbandona. 


\section{CANTO QUINTO}

Después que a mi doctor hube escuchado nombrar damas de ayer $y$ caballeros de compasion sentime quebrantado.

$Y$ le dije: -Poeta, placentero hablaria a esos dos que van unidos $y$ que el viento arrebata tan ligeros.

$Y$ él me repuso: -Cuando sean venidos más cerca, en nombre de su amor les ruega $y$ verás cómo vienen complacidos.

Tan pronto como el viento las allega les proferi: $-i$ Oh almas angustiadas venid a hablarnos si alguien no os lo niega!

Como palomas del deseo llamadas con ala abierta y firme al dulce nido surcan el aire del querer llevadas.

Asi dejaron la escuadra de Dido a nuestro encuentro en el airc maligno, que el reclamo de amor fué tan sentido.

-iOh tú, mortal gracioso y benigno que visitas enmedio el fosco cierzo a los que al mundo dimos cruento signo!

Si amigo fuese el rey del universo le rogariamos te diese paz, ya que te apiadas de este mal perverso.

De lo que oir y hablar ie da solaz nos placerenos en hablar $y$ oir mientra enmudece el viento lenguaraz.

Yace la tierra do empecé a vivir al pie del mar en donde el Po desciende para con sus tributarios dormir.

Amor que el noble corazón enciende, a éste encendió por la bella persona que me fue rapta en son que auin me ofende.

Amor que al ser amado no perdona que ame, de este placer me asio tan fuerte que, como ves, aun no me abandona. 
Amor condusse noi ad una morte; Caina attende chi'n vita ci spense. Queste parole da lor ci fur porte.

Da ch'io'ntesi qell'anime offense chinai'l viso e tanto'l tenni basso fin ch'il poeta mi disse: -Che pense?

Quando risposi comminciai: -Oh lasso, quanti dolci pensier, quanto disio meno costoro al doloroso passo:

Poi mi rivolsi a loro e parlai io, e comminciai: -Franccsca, $i$ tuoi martiri

a lagrimar mi fonno tristo e pio.

Ma dimmi: al tempo de'dolci sospiri a che e come concedetile Amore che conesceste $i$ dubbiosi desiri?

Ed ella a me: -Nessun maggior dolore che ricordarsi dal lempo felice nella miseria; e ció sa'l tuo dottore.

Ma se a conoscer la prima radice del nostro amor tu hai cotanto affetto, diró come colui che piange $e$ dice.

Noi leggevamoGu giorno per dilet to di Lancilotto come Amor lo strinse: soli eravamo esenza alcun sospetto.

Per piú fiate gli occhi ci sospinse quella lettura e scolorocci'l viso; ma solo un punto fu quel che ci vinse.

Quando leggemo il disiato riso esser baciato di cotanto amante, questi che mai da me non fia diviso.

La bocca mi bació tutto tremante;

Galeotto fu il libro e chi lo scrisse: Quel giorno pia non vi legemmo avante.

Mentre che l'uno spirto questo disse l'altro piangeva si che di pietade io venni men cosi com'io morisse, e caddi come corpo morte cade. 
Condújonos Amor a una muerte.

Caina aguarda al autor de esta ofensa.

$\mathrm{Su}$ voz nos trajo el viento de esta suerte.

Cuando entendi su aflicción inmensa

incliné el rostro $y$ tanto fui agobiado

hasta que el vate me dijo: - ¿En qué piensas?

$Y$ yo le respondi: $-i A y$, desdichados,

$q u e ́$ deseos, qué dulces pensamientos

los condujeron al doliente estado!

Luego, tornando a ellos mis acentos

dije: - Francisca, triste y compasivo,

me hacen derramar llanto tus tormentos.

Mas dime, al tiempo del suspiro vivo,

¿en qué manera concedió el Amor

que conociérais el deseo furtivo?

$Y$ ella repuso:- No hay mayor dolor

que recordar las horas de ventura

en las de mal. Lo sabe tu Doctor.

Mas si de nuestro amor con tal ternura

la primera raiz quieres saber

llorando te diré su desventura.

Leiamos un día por placer

como de Lanzarote Amor fué aueño,

solos $y$ sin acechanzas temer.

Varias veces aquel texto halagüeño

suspendimos, la faz desencajada;

mas solo un punto venció nuestro ensueño.

Al leer que la sonrisa codiciāa

de la boca besara el fiel amante

éste de quien jamás seré apartada,

Besó la mia, trémulo, anhelante.

Galeoto fué el libro $y$ su escritor.

Más no leimos desde aquel instante.

Mientras asi narraba su dolor

un espiritu, el otro tal plañia

que me invadió un desmayo abrumador

y cai cual cadáver caería.

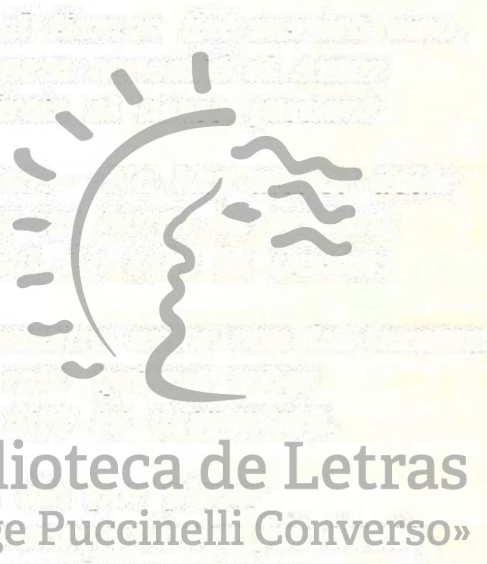


La bocca sollevó dal fiero pasto quel peccator, forbendola a'capelli del capo ch'egli avea diretro guasto;

Poi comminció: -Tu vuoi ch'io rinnovelli disperato dolor chel cuor mi preme giá pur pensando pria ch'io ne favelii.

Ma se le mie paro'e esser den seme che frufti infamid al traditor chio rodo, parlare e lagrimar mi vedrai insieme.

Io non so chi tu se, ne per che modo venuto se'quaggiai; mu Fiorentino mi sembri veramente quad'io t'odo.

$T u$ dei saper ch'io fui'l Conte Ugolino, $e$ questi l'arcivescovo Ruggieri:

or ti diró percỉ'i son tal vicino.

Che per l'effetto de'suoi ma'pensieri, fidandomi di lui, io fossi preso e poscia morto, dir non è mestieri.

Peró quel che non puoi aver inteso, cio é come la morte mia fu cruda, udirai, e saprai se m'ha offeso.

Brieve pertugio dentro dalla Muda, la qual per me ha il titol aclla jame, $e^{\prime} n$ che conviene ancor ch'altri si chiuda.

M'avea mostrato per lo suo forame piú lune già guand'io fece'l mal sonno che del futuro mi squarciól velame. Questi pareva a me maestro e donno, caccianclo il lupo e $i$ lupicini al monte perch'ii Pisan veder Lucca non ponno,

Con cane magre studiose $e$ conte;

Gualandi con Sismondi $c$ con Lanfranchi s'avea messi dinanzi dalla fronte.

In picciol corso mi pareano stanchi lo padre $e$ i figli, e con l'agute scane mi parea lor veder fender li fianchi. 
$L A$ boca retiró del vil bocado el pecador, limpiándola en el pelo del cráneo que royera encarnizado;

$Y$ comenzó: - Quieres que el desconsuelo renueve del dolor que me lacera solo al pensarlo, sin decir tal duelo;

Pero si mi valabra ser pudiera valdón para el traidor a quien devoro llorando te diré lo que ocurriera.

Quién eres ni por qué vienes lo ignoro, mas perécesme, cierto, Florentino cuando hablar te oigo en la lengua que añoro

Haz de saber que fui el conde Ugolino $y$ este es el arzobispo Ruggiero: por qué soyle, verás, tan cruel vecino.

Huelga decir que por su genio fiero y mi confianza en él fuई aprisionado $y$ después muerto, cuando prisionero.

Mas lo que nunca te habrán relatado, quiero decir, la crueldad de mi muerte, oirds y sabrás si me ha agraviado.

Breve Thendiduracen ala torreldel fuerte, que es la del Hambre hoy, por mi vejamen, $y$ donde seguirán otros mi suerte.

Me habia mostrado al través su foramen ya varias lunas cuando hube el mal sueño que del futuro me rasgó el velamen.

Este se me mostró señor $y$ dueño, lobo y lobeznos cazando en el cerro que oculta Lucca a Pisa, zahareño,

con jauria de magros, fieros perros. Los Lanfrancos, Sismondos y Gualandos iban delante de él por aquel cerro.

Tras breve cabalgata vi jadeando al padre e hijos e hincarles los dientes los canes, sus costados desgarrando. 
Quando fui desto innanzi la dimane pianger senti'fra'l sonno $i$ miei figliouoli ch'erano meco, e dimandar del pane.

Ben se crudel se tu giá non ti duoli pensanclo ció ch'al mio cuor si annunziava: $e$ se non piangi, di che pianger suoli?

Giá eran desti e l'ora s'appressava ch'l cibo ne soleva esscr addotto, $e$ per suo sogno ciascun dubitava.

$E d$ io senti'chiavar l'uscio di sotto all'orribile torre; ond'io guardai nel viso a'miei figliol senza far motto.

Io non piangeva, si dentro impietrai; piangevan elli, ed Anselmuccio mio disse: - Tu guardi sí, padre, che hai?

Peró non lagrimai ne rispos'io tutto quel giorno ne la notte apresso, infin che l'altro sol nel mondo uscio.

Come un poco di raggio si fu messo nel doloroso carcere, ed io scorsi per quatro visi il mio aspletto stesso.

Ambo le mani di dolor mi morsi: e quei pensando crio'i fessi per vogtias di manicar di subito levorsi.

$E$ disser: - Padre, assai ci fia men doglia se tu mangi di noi; tu ne vestisti queste misere carni, e tu le spoglia

Quetá mi allor per non farli piu tristi; quel di a l'altro stemmo tutti muti; Ahi dura terra, perche non t'apristi?

Poscia che fummo al quarto di venuti Gaddo mi si gittó disteso ai piedi dicendo: -Padre mio, che non m'ajuti?

Quivi mori; e come tu mi vedi vid'io cascar li tre ad uno ad uno tra'l quinto di e il sesto; ond'io mi diedt

Giá cieco a brancolar sovra ciascuno, $E$ due di li chiamai poi che fur morti; Poscia piu che'l dolor poté il digiuno. 
Antes del alba al despertar, dolientes, a mis hijos of en sueños llorar pidiendome, a mi lado, pan, yacentes.

Muy cruel serás, si no te hace apiadar lo que mi corazón ya sospechaba; y si no lloras, ¿qué te hará llorar?

Ya despiertos, la hora se acercaba en que solian traernos la comida, mas por su sucño cada cual dudaba.

$Y$ of clavar la puerta de salida de la horrida torre, por lo cual miré a mis hijos con mudez transida.

Yo no lloraba, vuelto pedernal; ellos si y Anselmito dijo asi; - Tu mirar, padre, ¿presagia algûn mal?

$\mathrm{Ni}$ lágrimas vertí ni respondi el dia aquel ni la noche siguiente hasta que un nuevo sol alumbrar vf. Cuando ilumino un rayo débilmente la carcel dolorosa y sus semblantes me reflejaron mi expresion doliente, Mordf mis manos, de dolor tremante, y ellos lcreyendo que lo hacia de hambriento alzâronse y dijeronme al instante:

- Padre, menos será nuestro tormento si comes nuestra carne; nos vestiste con ella; que ahora sea tu alimento.

Callé para no verlos más tristes. Dos dias más nuestra prisión fué muda. ‘Ay, dura tierra! ¿Por qué no te abriste?

Al despuntar la cuarta aurora cruda Gaddo, diciendo, se tendio a mis pies:

-Padre, ¿por qué no me prestas tu ayuda?

Allí murío, $y$ así como me ves ví caer a los tres uno por uno, del quinto al sexto día. Y ya después

Ciego, me eché a palpar a cada uno, $y$ muertos los llamé tres días, tres; luego, mas que el dolor pudo el ayuno. 